\title{
Pre-classical solutions of the vacuum Bianchi I loop quantum cosmology
}

\author{
Ghanashyam Date* \\ The Institute of Mathematical Sciences, CIT Campus, Chennai-600 113, INDIA
}

\begin{abstract}
Loop quantization of diagonalized Bianchi class A models, leads to a partial difference equation as the Hamiltonian constraint at the quantum level. A criterion for testing a viable semiclassical limit has been formulated in terms of existence of the so-called pre-classical solutions. We demonstrate the existence of pre-classical solutions of the quantum equation for the vacuum Bianchi I model. All these solutions avoid the classical singularity at vanishing volume.

PACS numbers: 04.60.Pp,98.80.Jk,98.80.Bp
\end{abstract}

Loop Quantum Gravity (LQG) is the leading candidate for a manifestly background independent approach to constructing a quantum theory of gravity [1]. This approach is particularly well suited in the context where the Einstein theory indicates occurrence of singularities entailing highly dynamical geometries with extreme curvatures. The methods employed in this approach can be adapted and tested in the simpler context of cosmological models. Quantizing the cosmological models along the lines of LQG has lead to the development of Loop Quantum Cosmology (LQC) 2].

One of the crucial simplification available in LQC is the existence of the triad representation and knowledge of complete spectrum of the volume operator so crucial for quantization of the Hamiltonian constraint. The fact that the holonomies of the connection are well defined operators but not the connection it self, is directly responsible for the two main features of LQC: (a) the quantum Hamiltonian constraint leads to a difference equation 3] and (b) inverses of triad components have bounded spectra [4, 5]. Both these features lead to the absence of 'singularities' in the quantum theory [6, 7, 8,9$]$ ].

While loop quantization of cosmological models is well specified, one also needs to check if the quantum dynamics admits solutions (semiclassical states) which can approximate the classical description. A natural way to recover classical behavior would be in terms of the expectation values of suitable observables in the semiclassical states. LQC (and LQG) being constrained systems, makes such a recovery of classical behavior, more complicated. The solutions of the Hamiltonian constraint, the only relevant one for LQC, are typically distributional and one needs to equip the space of solutions with a new (physical) inner product. One also needs to identify suitable (Dirac) observables. Addressing these aspects is at a preliminary stage 10, 11, 12, 13.

Current understanding of the semiclassical limit of LQC is centered around the notion of pre-classicality. The articulation of this notion has undergone a few changes and it is useful to note them. Originally proposed in 14], pre-classicality was thought in terms of obtaining the continuum Wheeler-De Witt equation from the fundamental difference equation by a limit in which the Barbero-Immirzi parameter is taken to zero. Sub- sequently, the idea of pre-classical limit was replaced by the idea of a pre-classical approximation 15. To obtain (modified) Einstein dynamics from the Hamilton-Jacobi equation provided by a WKB approximation 16, 17, 18], it was shown to be sufficient to have approximate preclassical solution(s) with a finite domain of validity. The property of local stability is needed in the construction of such solutions [15] and is satisfied by difference equations of LQC [9]. Recently, the methods of analyzing the asymptotic behavior of exact solutions of the difference equations have been developed [19, 20] which provide the sharpest yet formulation of pre-classicality of a solution [19]. Briefly, the criterion is that asymptotically, small scale oscillations in the solution be suppressed.

Pre-classical solutions so identified are known to exist for isotropic models $[6,14,20]$ and some of the LRS models [19]. However, for the anisotropic, vacuum Bianchi I model, pre-classical solutions were shown not to exist 21]. While this result is true for solutions that pass through vanishing volume, there are more possibilities which permit existence of pre-classical solutions. Since the works in [8, 9], the so called Bohr quantization has been developed [7] which is crucial for the existence of pre-classical solutions and we incorporate it in the brief summary of the quantum theory given below.

The kinematical Hilbert space is spanned by orthonormalized vectors labeled as $\left|\mu_{1}, \mu_{2}, \mu_{3}\right\rangle, \mu_{I} \in \mathbb{R}$. These are properly normalized eigenvectors of the triad operators $p^{I}$ with eigenvalues $\frac{1}{2} \gamma \ell_{\mathrm{P}}^{2} \mu_{I}$, where $\gamma$ is the BarberoImmirzi parameter and $\ell_{\mathrm{P}}^{2}:=8 \pi G \hbar:=\kappa \hbar$. The volume operator is also diagonal in these labels with eigenvalues $V(\vec{\mu})$ given by $\left(\frac{1}{2} \gamma \ell_{\mathrm{P}}^{2}\right)^{3 / 2} \sqrt{\left|\mu_{1} \mu_{2} \mu_{3}\right|}$. Here we have used the vector notation to denote the triple $\left(\mu_{1}, \mu_{2}, \mu_{3}\right)$. Imposing the Hamiltonian constraint operator on general vectors of the form $|s\rangle=\sum_{\vec{\mu}} s(\vec{\mu})|\vec{\mu}\rangle$ leads to the fundamental difference equation for the wave function $s(\vec{\mu})$. Here the sum is over countable subsets of $\mathbb{R}^{3}$. The wave function $s(\vec{\mu})$ have to be invariant under simultaneous reversal of signs of a pair of $\mu_{I}$ 's and corresponds to the residual freedom of reversing the sign of any two of the triad components [8].

In the present context of vacuum Bianchi I model, the 
Hamiltonian constraint leads to the equation [9],

$$
\sum_{\vec{\epsilon}_{12}} A_{12}\left(\vec{\mu} ; \vec{\epsilon}_{12}\right) s\left(\vec{\mu} ; \vec{\epsilon}_{12}\right)+\text { cyclic }=0, \text { where }
$$

$\vec{\epsilon}_{12}=\left(\epsilon_{1}, \epsilon_{2}, \epsilon_{1}^{\prime}, \epsilon_{2}^{\prime}\right)$ with each of the $\epsilon_{*}$ taking values \pm 1 ; $s\left(\vec{\mu} ; \vec{\epsilon}_{12}\right)=s\left(\mu_{1}-\mu_{0} \epsilon_{1}-\mu_{0} \epsilon_{1}^{\prime}, \mu_{2}-\mu_{0} \epsilon_{2}-\mu_{0} \epsilon_{2}^{\prime}, \mu_{3}\right) ; \mu_{0}$ is an order 1 parameter and,

$$
\begin{aligned}
A_{12}\left(\vec{\mu} ; \vec{\epsilon}_{12}\right) & =V\left(\vec{\mu} ; \vec{\epsilon}_{12}\right) d\left(\mu_{3}\right)\left(\epsilon_{1} \epsilon_{2}+\epsilon_{1}^{\prime} \epsilon_{2}^{\prime}\right) \\
d(\mu) & :=\left\{\begin{array}{cc}
\left|1+\mu_{0} \mu^{-1}\right|^{\frac{1}{2}}-\left|1-\mu_{0} \mu^{-1}\right|^{\frac{1}{2}} & \mu \neq 0 \\
0 & \mu=0
\end{array}\right.
\end{aligned}
$$

Note that while the equation (11) is defined for all $\mu_{I} \in \mathbb{R}$, it is actually a difference equation since only the coefficients $s(\vec{\mu})$, differing in steps of $\pm 2 \mu_{0}$ are constrained by the equation. One can make this explicit by setting $\mu_{I}:=2 \mu_{0} \nu_{I}+2 \mu_{0} N_{I}, N_{I} \in \mathbb{Z}$ and $S_{\vec{N}}(\vec{\nu}):=s(\vec{\mu})$. Clearly, there are infinitely many 'sectors' labeled by $\vec{\nu}$ with $\nu_{I} \in[0,1)$. Only those sectors for which at least one of the $\nu_{I}$ is zero, one will encounter zero volume.

It is convenient to absorb the factors of volume eigenvalues into the wave functions by defining $t(\vec{\mu}):=$ $V(\vec{\mu}) s(\vec{\mu})$ so that the equation (11) becomes,

$$
\sum_{\vec{\epsilon}_{12}} d\left(\mu_{3}\right)\left(\epsilon_{1} \epsilon_{2}+\epsilon_{1}^{\prime} \epsilon_{2}^{\prime}\right) t\left(\vec{\mu} ; \vec{\epsilon}_{12}\right)+\text { cyclic }=0
$$

This preserves the gauge invariance condition on the $s(\vec{\mu})$. Furthermore, due to the explicit volume eigenvalues, $t_{\mu_{1}, \mu_{2}, \mu_{3}}=0$ if any of the $\mu_{I}$ 's equal zero.

Making a product ansatz for the wave function and introducing the difference operator $\Delta$,

$$
\begin{aligned}
t(\vec{\mu}) & :=z_{1}\left(\mu_{1}\right) z_{2}\left(\mu_{2}\right) z_{3}\left(\mu_{3}\right), \\
\Delta z_{I}\left(\mu_{I}\right) & :=\left\{z_{I}\left(\mu_{I}+2 \mu_{0}\right)-z_{I}\left(\mu_{I}-2 \mu_{0}\right)\right\},
\end{aligned}
$$

the difference equation (3) can be written as,

$$
d\left(\mu_{3}\right) \Delta z_{1}\left(\mu_{1}\right) \Delta z_{2}\left(\mu_{2}\right) z_{3}\left(\mu_{3}\right)+\text { cyclic }=0 .
$$

The gauge invariance conditions then translate to $z_{I}\left(-\mu_{I}\right)=\eta z_{I}\left(\mu_{I}\right), \forall I$, where $\eta= \pm 1$. The vanishing of $t(\vec{\mu})$ when any of the $\mu_{I}=0$, translates into the condition: $z_{I}(0)=0, \forall I$.

An exact solution of the partial difference equation can be obtained by setting,

$$
\Delta z_{I}\left(\mu_{I}\right)=\beta_{I} d\left(\mu_{I}\right) z_{I}\left(\mu_{I}\right), \quad \forall I, \quad \forall \mu_{I} \in \mathbb{R}
$$

where $\beta_{I}$ are some constants which have to satisfy,

$$
\beta_{1} \beta_{2}+\beta_{2} \beta_{3}+\beta_{3} \beta_{1}=0=\left(\sum_{I} \beta_{I}\right)^{2}-\sum_{I} \beta_{I}^{2} .
$$

Thus we obtain a class of exact solutions of the partial difference equation (3) from those of three, ordinary difference equations (7) with parameters $\beta_{I}$ satisfying (8).
The original partial difference eq. (11) is linear and with real coefficients, $A_{I J}$ 's. So, without loss of generality, we can assume the wave function, $s(\vec{\mu})$ to be real. Since for the product ansatz, all $z_{I}$ are independent, these must be real as well which requires that the $\beta_{I}$ 's be real.

If all $\beta_{I}$ are zero, then $z_{I}$ 's are constants and so is $t(\vec{\mu})$. These cannot satisfy the condition $z_{I}(0)=0$ without making $t(\vec{\mu})=0$ identically. The non-trivial solutions then cannot pass through zero volume and must belong to the sectors with $\nu_{I} \neq 0$. This also shows that in the sectors with $\nu_{I} \neq 0, \forall I$, there is an exact pre-classical solution to (II) namely $t\left(2 \mu_{0} \vec{\nu}+2 \mu_{0} \vec{N}\right)=\operatorname{constant}(\neq$ $0), \forall \vec{N} \in \mathbb{Z}^{3}$.

If at least one $\beta_{I} \neq 0$, then we can always take out a common factor from all $\beta_{I}$ and ensure $\sum_{I} \beta_{I}=1$. Equivalently, a common scaling of $\beta_{I}$ can be absorbed by a common inverse scaling of the $d(\mu)$ functions, which amounts to a scaling of the volume which cancels out in (11). The class of solutions that is being constructed can thus be parameterized exactly in the same manner as the classical Kasner solution. In particular either two of the $\beta$ 's are zero or exactly one is negative while other two are positive. From now on we will restrict to $0<\left|\beta_{I}\right|<1$.

To explore pre-classicality of the separable solution, let us focus on (7), suppressing the label $I$. Introducing the notation: $\mu:=2 \mu_{0} \nu+2 \mu_{0} n, n \in \mathbb{Z}, \nu \in[0,1)$ and $z(\mu):=Z_{n}^{(\nu)}$, (7) can be written as:

$$
\begin{aligned}
Z_{n+2}^{\nu} & =Z_{n}^{\nu}+\beta d(\nu, n+1) Z_{n+1}^{\nu} \quad, \quad \forall n \in \mathbb{Z} \\
d(\nu, n) & :=\left|1+\frac{1}{2(\nu+n)}\right|^{\frac{1}{2}}-\left|1-\frac{1}{2(\nu+n)}\right|^{\frac{1}{2}}
\end{aligned}
$$

We have infinitely many decoupled sectors, labeled by $\nu$ and for each of these we have a second order, ordinary difference equation. Due to linearity, only one condition is enough to determine a solution. Only for the sector $\nu=$ 0 ( $\mu$ is integer multiple of $2 \mu_{0}$ ), the condition $z(0)=0$ is relevant and it fixes the solution completely. The gauge invariance condition translates into the identification:

$$
Z_{-(n+1)}^{1-\nu}=\eta Z_{n}^{\nu}, \quad \forall n \in \mathbb{Z}, \quad \eta= \pm 1
$$

which restricts the $Z$ 's in the same sector, only for $\nu=0, \frac{1}{2}$. For all other sectors the gauge invariance condition relates two different sectors. Under the identification implied by (10), the equation satisfied by $Z_{n}^{\nu}$ goes over to the equation satisfied by $Z_{n}^{1-\nu}$ automatically with the same value of $\beta$. Therefore the solutions in the sector $(1-\nu)$ can all be obtained from solutions in the sector $\nu$ via (10). For $\nu=\frac{1}{2}$, the gauge invariance condition requires $Z_{-1}^{1 / 2}=\eta Z_{0}^{1 / 2}$ which fixes the solution completely. For the two sectors, $\nu=0, \frac{1}{2}$, pre-classicality is not optional - the solution is either pre-classical or it is not. From now on the superscript $\nu$ is suppressed.

Consider (9). Defining the ratios $u_{n+1}:=Z_{n+1} / Z_{n}$, one can see that $\left[\left(u_{n+2}-\beta d(\nu, n+1)\right) u_{n+1}-1\right] Z_{n}=0$. 
There are several possibilities now ( $n \geq 0$ for definiteness). If $Z_{n}$ remains non-zero for all $n$, then $u_{n} \rightarrow \pm 1$ as $n \rightarrow \infty$. The $u_{n} \rightarrow-1$ is referred to as a sequence with sign oscillations. Since $u_{n+2}-\beta d(\nu, n+1)$ and $u_{n+1}$, must have the same sign, it is clear that if $u_{1}>0$ and $\beta>0$, then $u_{n}>0$ for all $n$. For $\beta<0$ however, $u_{n}$ may become negative for some $n_{0}$ and then stay negative subsequently. Whether this could happen depends on the value of $u_{1}$. Thus it is conceivable that for some positive values of $u_{1}$, one could have a sequence without sign oscillations. If $Z_{n}$ converges to zero, then $\left|u_{n}\right|$ converges to a value $\leq 1$, once again allowing for $u_{n} \rightarrow-1$. The sequence $u_{n}$ could also converge to 0 in which case there could be oscillations about 0 , but these are suppressed. By pre-classicality we mean either absence or suppression of sign oscillations.

To identify pre-classical sequences, we employ the generating function technique [19]. The function $d(\mu)$ being an algebraic function poses some difficulties which can be handled in exactly the same way as in [19]. Basically, one separates out the large $n$ part of $d(\nu, n)$ and solves the equation perturbatively, $Z_{n}=a_{n}+\sum_{k=1}^{\infty} Z_{n}^{k}$. The leading order term, $a_{n}$ satisfies the equation with $d(\nu, n)$ replaced by $(2(n+\nu))^{-1}$. Let us focus on $n \geq 0$ so that the absolute signs can be removed. Setting $\beta=4 \lambda$ the equation defining the $a_{n}$ sequence is,

$$
a_{n+2}-\frac{2 \lambda}{n+1+\nu} a_{n+1}-a_{n}=0, n \geq 0 .
$$

To account for non-integral $\nu$, we define a generating function $F(x):=\sum_{n=0}^{\infty} a_{n} x^{n+\nu}$ and the function $G(x):=$ $x^{-1}\left(F(x)-a_{0} x^{\nu}\right)$, which satisfies a differential equation equivalent to the difference equation (11),

$$
\frac{d}{d x}\left(\left(1-x^{2}\right) G\right)-2 \lambda G-a_{0}(\nu+1) x^{\nu}-a_{1} \nu x^{\nu-1}=0
$$

For non-zero $\nu$, the last term is singular at $x=0$ implying that $G(x)$ will not be analytic at $x=0$. However, the singular term is integrable such that $G(x)$ is continuous at $x=0$. The series representation requires it to vanish at $x=0$.

The equation (12) can be easily integrated to give,

$$
\begin{aligned}
G(x)= & (1+x)^{\lambda-1}(1-x)^{-\lambda-1}\left[c_{0}+\right. \\
& \left.\int^{x}\left(\frac{1-t}{1+t}\right)^{\lambda}\left\{a_{0}(1+\nu) t^{\nu}+a_{1} \nu t^{\nu-1}\right\}\right]
\end{aligned}
$$

Notice that given any $a_{0}, a_{1}$, the solution to (11) is completely determined and so should be $G(x)$. The above solution for $G(x)$ contains an indefinite integral and an arbitrary constant of integration, $c_{0}$. We must choose $c_{0}$ and convert the indefinite integral to a definite one such that $G(x)$ corresponds to the sequence specified by the given $a_{0}, a_{1}$. The only value of $G(x)$ we know without having to know the full sequence is $G(0)=0$. Furthermore both the integrands in the integrals in (13) are integrable at $x=0$. Thus it is possible to impose $G(0)=0$ which then requires $c_{0}=0$. The generating function is then obtained as,

$$
\begin{aligned}
(1-x) G(x)= & (1+x)^{\lambda-1}(1-x)^{-\lambda} \times \\
& {\left[a_{0} I(\nu, \lambda, x)+a_{1} I(\nu-1, \lambda, x)\right], } \\
I(\nu, \lambda, x):= & (1+\nu) \int_{0}^{x}\left(\frac{1-t}{1+t}\right)^{\lambda} t^{\nu}
\end{aligned}
$$

One may already note that the integrals are finite at both $x= \pm 1$ but the pre-factor is not. Thus singularities of $(1-x) G(x)$ are controlled by the pre-factor which is independent of $\nu$. Apart from the $\nu$ dependence of the integrals, one does not expect qualitative behavior of $G(x)$ to be affected by $\nu$. Furthermore, since there are two free parameters $\left(a_{0}, a_{1}\right)$ which specify the sequence and only one of these is relevant one due to the linearity, one can at the most impose only one condition capturing pre-classicality, to get a non-trivial solution.

Now consider the behavior of $G(x)$ as $x \rightarrow-1$. For the range of $0<|\lambda|<1$, both the integrals exist (and are positive), but the pre-factor diverges. A divergence in $G(x)$ at $x=-1$ implies un-suppressed sign oscillations which are to be avoided for pre-classical sequences [19]. Clearly, to avoid this singularity in $G(x)$, the integrals must add up to zero which determines $a_{1}$ in terms of $a_{0}$. For $x=-1$, one has,

$$
\begin{aligned}
I(\nu, \lambda,-1)= & (-1)^{\nu} I(\nu,-\lambda, 1) \\
I(\nu,-\lambda, 1)= & (1+\nu) B(1+\nu, 1-\lambda) \times \\
& F(-\lambda, \nu+1,-\lambda+\nu+2 ;-1),
\end{aligned}
$$

where, $B$ and $F$ are the Beta function and the hypergeometric functions [22]. The condition of no singularity at $x=-1$ gives,

$$
a_{0} I(\nu,-\lambda, 1)=a_{1} I(\nu-1,-\lambda, 1) .
$$

This determines the sequence uniquely modulo a trivial, overall scaling. The sequence satisfying (18) has suppressed sign oscillations. Its convergence properties are determined by the $x \rightarrow 1$ behavior of $(1-x) G(x)$. For $x=1$ the integrals again exist but now the pre-factor diverges for $\lambda>0$ and vanishes for $\lambda<0$ and so does the sequence $\left\{a_{n}\right\}$. However, $(1-x) G(x)$ is integrable at $x=1$ which implies that the asymptotic behavior of $a_{n}$ is bounded by $n$. Making a power law ansatz for asymptotic $a_{n}$, one can see from (11) (and indeed from (9) as well) that $a_{n} \sim n^{\lambda}$. Since $\beta_{I}$ come with both signs, both behaviors must be admissible. For $\lambda=0$, one gets $a_{1}=a_{0}$ and the sequence is the constant sequence $a_{n}=a_{0} \forall n \geq 0$ which is obviously pre-classical.

As anticipated, these results are exactly analogous to those obtained in [19]. Indeed, for $\nu=0$, equations (11) 12 13) go over to the equations of [19]. Now the boundary condition is $G(0)=a_{1}$ which gives $c_{0}=a_{1}$ and definite integral has the lower limit as 0 . Demanding nonsingularity of $G(-1)$, determines $a_{1}$ exactly as in [19]. 
The behavior at $x=1$ is similar to that for the non-zero $\nu$ case.

Thus, there certainly exist sectors such that for each choice of the separation constant $\beta, 0<|\beta|<1$, one can select $a$ unique solution of (11) which is pre-classical. These solutions of course have to be improved by computing the corrections $Z_{n}^{k}$ [19]. The asymptotic power law behavior of $a_{n}$ will continue to hold also for $Z_{n}$.

All these statements hold for $n \geq 0$. Having determined $Z_{0}^{\nu}, Z_{1}^{\nu}$, by pre-classicality, $Z_{n<0}$ can be determined by the exact equation (9). Whether $Z_{n}^{\nu}$ is preclassical also for $n<0$, can be inferred by testing for pre-classicality of $Z_{n}^{1-\nu}$ for positive $n$, using (10).

As noted earlier, the sectors $\nu=0, \frac{1}{2}$ already have a unique solution due to the conditions $Z_{0}^{0}=0$ and $Z_{-1}^{1 / 2}=$ $\eta Z_{0}^{1 / 2}$ respectively. If these conditions are imposed on the leading pre-classical sequence $\left\{a_{n}\right\}$, then clearly there are no non-trivial solutions in the $\nu=0$ sector. For $\nu=1 / 2$ sector, numerically, the gauge invariance condition and the pre-classicality condition seem to hold only for $\beta=0$ with $\eta=1$. For other sectors, pre-classicality is the only condition imposed and solutions can be constructed.

The full wave function is the product of the three sequences and apart from an over all constant factor, is completely determined. One can build more general (and non-separable) solutions by taking complex linear combinations with coefficients being functions of $\vec{\beta}$. Clearly, a combination involving a diverging and a vanishing solution will be a diverging one and still without sign oscillations. Since such a solution involves a distribution of $\vec{\beta}$, these parameters themselves would not be identified with the classical Kasner parameters, $\alpha_{I}$ (say) satisfying $\sum_{I} \alpha_{I}=1=\sum_{I} \alpha_{I}^{2}$. Rather, one would imagine constructing linear combinations which 'peak' in some suitable sense, around three triad values $p_{0}^{I}$ and a Kasner parameter $\vec{\alpha}_{0}$. (Since the reduced phase space of the vacuum Bianchi I model is $4=6-2$ dimensional, one needs four parameters to specify a classical solution and these could be conveniently taken as three initial triad values and a Kasner parameter value.) If such a construction can be carried out, then one would be able to claim that the quantum theory has 'sufficient number of semiclassical solutions' as expected from the classical theory.

We note that since solutions of the Hamiltonian constraint are expected to be distributional in general, kinematical normalizability of the pre-classical (or otherwise) solutions is not directly mandated. The requirement of pre-classicality for both signs of $n$ is an open issue. For an alternative treatment of separable solutions, see [24].

In summary, we have shown that in every sector $\nu_{I} \neq$ $0, \frac{1}{2}$, there exist a one parameter family of pre-classical solutions. For $\beta_{I}=0$, the solution is in fact exact, possibly corresponding to the Minkowski space-time. All these solutions skip the vanishing volume eigenvalues. By contrast, in the $\nu_{I}=0$ sector, there are no pre-classical solutions 21]. The richness of the loop quantization, man- ifested by the infinitely many sectors, is crucial for this result; an observation also made in 11] in the isotropic context. We have heuristically indicated how these families can be used to see if loop quantization does admit 'enough semiclassical states'. The exact, non-singular solutions of the effective dynamics of vacuum Bianchi I models given in 23. also exhibit a similar feature of avoiding vanishing volume which motivated this work.

I would like to thank Martin Bojowald for helpful remarks particularly regarding vanishing and diverging sequences vis a vis pre-classicality. Discussions with Golam Hossain are also acknowledged.

* Electronic address: shyam@imsc.res.in

[1] C. Rovelli, Living Reviews in Relativity 1, (1998) 1; T. Thiemann, Lect. Notes Phys. 631, (2003) 41; A. Ashtekar and J. Lewandowski, Class. Quant. Grav. 21, (2004) R53.

[2] Bojowald M and Morales-Técotl H A 2004 Cosmological applications of loop quantum gravity, Lect. Notes Phys., 646, Ed. Bretón, N , Cervantes-Cota, J and Salgado, M, Springer-Verlag.

[3] M. Bojowald, Class. Quantum Grav. 18, (2001) 1055; M. Bojowald, Class. Quantum Grav. 18, (2001) 1071.

[4] M. Bojowald, Phys. Rev. D 64, (2001) 084018.

[5] M. Bojowald, Pramana 63, (2004) 765.

[6] M. Bojowald, Phys. Rev. Lett. 86 (2001) 5227.

[7] A. Ashtekar, M. Bojowald and J. Lewandowski, Adv. Theor. Math. Phys. 7, (2003) 233.

[8] M. Bojowald, Class. Quantum Grav. 20, (2003) 2595.

[9] M. Bojowald, G. Date, and K. Vandersloot, Class. Quantum Grav. 21, (2004) 1253.

[10] G. M. Hossain, Class. Quantum Grav, 21, (2004), 179.

[11] K. Noui, A. Perez and K. Vandersloot, Phys. Rev. D 71, (2005) 044025.

[12] B. Dittrich, Partial and complete observables for Hamiltonian constrained systems, gr-qc/0411013.

[13] A. Ashtekar, L. Bombelli and A. Corichi, Phys.Rev. D 72 (2005), 025008

[14] M. Bojowald, Phys. Rev. Lett. 87, (2001) 121301.

[15] M. Bojowald and G. Date, Class. Quantum Grav. 21, (2004) 121.

[16] M. Bojowald, Class. Quantum Grav. 18, (2001) L109.

[17] G. Date and G. M. Hossain, Class. Quantum Grav., 21, (2004) 4941.

[18] K. Banerjee and G. Date, Class. Quantum Grav, (to appear), 2005.

[19] D. Cartin, G. Khanna and M. Bojowald, Class. Quantum Grav. 21, (2004) 4495.

[20] M. Bojowald and A. Rej, Class. Quantum Grav. 22, (2005) 3399.

[21] D. Cartin and G. Khanna, Phys. Rev. Lett. 94, (2005) 111302.

[22] Handbook of Mathematical Functions, Ed. M. Abramowitz and I. A. Stegun, Dover Publ. Inc., New York, 1972.

[23] G. Date, Phys. Rev. D 71, (2005) 127502.

[24] D. Cartin and G. Khanna, Separable wave functions in Bianchi I loop quantum cosmology, gr-qc/0506024. 